\title{
Treating childhood pneumonia in hard-to-reach areas: A model-based comparison of mobile clinics and community-based care
}

\author{
Catherine Pitt ${ }^{1 *}$, Bayard Roberts ${ }^{2}$ and Francesco Checchi $^{3}$
}

\begin{abstract}
Background: Where hard-to-access populations (such as those living in insecure areas) lack access to basic health services, relief agencies, donors, and ministries of health face a dilemma in selecting the most effective intervention strategy. This paper uses a decision mathematical model to estimate the relative effectiveness of two alternative strategies, mobile clinics and fixed community-based health services, for antibiotic treatment of childhood pneumonia, the world's leading cause of child mortality.

Methods: A "Markov cycle tree" cohort model was developed in Excel with Visual Basic to compare the number of deaths from pneumonia in children aged 1 to 59 months expected under three scenarios: 1) No curative services available, 2) Curative services provided by a highly-skilled but intermittent mobile clinic, and 3) Curative services provided by a low-skilled community health post. Parameter values were informed by literature and expert interviews. Probabilistic sensitivity analyses were conducted for several plausible scenarios.

Results: We estimated median pneumonia-specific under- 5 mortality rates of 0.51 ( $95 \%$ credible interval: 0.49 to 0.541 ) deaths per 10,000 child-days without treatment, 0.45 (95\% Cl: 0.43 to 0.48 ) with weekly mobile clinics, and 0.31 (95\% Cl: 0.29 to 0.32) with CHWs in fixed health posts. Sensitivity analyses found the fixed strategy superior, except when mobile clinics visited communities daily, where rates of care-seeking were substantially higher at mobile clinics than fixed posts, or where several variables simultaneously differed substantially from our baseline assumptions.
\end{abstract}

Conclusions: Current evidence does not support the hypothesis that mobile clinics are more effective than CHWs. A CHW strategy therefore warrants consideration in high-mortality, hard-to-access areas. Uncertainty remains, and parameter values may vary across contexts, but the model allows preliminary findings to be updated as new or context-specific evidence becomes available. Decision analytic modelling can guide needed field-based research efforts in hard-to-access areas and offer evidence-based insights for decision-makers.

Keywords: Pneumonia, treatment, mobile clinic, community health workers, decision model, crisis, humanitarian, remote, rural

\section{Background}

As of 2010, fewer than one-third of the 68 priority countries with the highest levels of child mortality were on track to meet the Fourth Millennium Development Goal (MDG) of reducing mortality in children aged under five by two-thirds between 1990 and 2015. In

\footnotetext{
* Correspondence: catherine.pitt@lshtm.ac.uk

'Department of Global Health \& Development, London School of Hygiene \&

Tropical Medicine, 15-17 Tavistock Place, London WC1H 9SH, UK

Full list of author information is available at the end of the article
}

many countries where overall progress has been made, that progress has been inequitable, with higher mortality among children in marginalised regions, especially remote areas and those affected by armed conflict [1,2]. In such settings, the leading causes of childhood deaths are pneumonia, diarrhoea, neonatal causes, and (where present) malaria [3-5].

Humanitarian relief agencies often provide services through mobile clinics to serve populations living beyond the reach of existing facilities. These mobile

\section{Biomed Central}


clinics often operate in chronic crises, where security is poor, logistics are difficult, and mortality is high. An increasing number of people affected by complex emergencies now live spread out across large and remote geographical areas, in places such as Somalia, Afghanistan, the Central African Republic, and Southern Sudan [3]. They are consequently harder to reach with humanitarian interventions and suffer prolonged excess mortality [3]. There is strong evidence that these mobile clinics, which usually provide relatively skilled services on an intermittent basis, can make a positive health impact with preventive activities such as vaccinations, hygiene promotion, and antenatal counselling, as well as through certain curative services for chronic or slowonset diseases such as intestinal parasites, guinea worm, leishmaniasis, onchocerciasis, and trypanosomiasis [6-9]. In many cases, however, mobile clinics also focus on curative care for rapid-onset illnesses, such as malaria, pneumonia, and diarrhoea [10].

There is also growing consensus around the effectiveness and safety of community-based management of pneumonia by supporting low-skilled health workers to provide oral antibiotics in low-income and remote areas [11-14]. A meta-analysis of nine trials in seven countries by Sazawal et al found that community antibiotic treatment strategies resulted in an average $24 \%$ reduction in total under-five mortality and a $36 \%$ reduction in pneumonia mortality in under-fives in high mortality settings [11]. In Nepal, one of the priority countries on track to meet MDG4 [2], female community health volunteers (FCHV) save an estimated 6000 child lives each year by providing oral antibiotics for non-severe pneumonia in remote mountain communities. Despite being only semi-literate, Nepal's FCHVs have shown "minimal" evidence of antibiotics misuse, and 30,007 case reviews in 2005-6 revealed "no evidence of emerging co-trimoxizole resistance" [12].

Despite this evidence, community-based management of pneumonia is not standard practice amongst relief agencies in less stable conflict-affected situations, and the most commonly used guidelines for humanitarian settings are designed primarily for physicians and nurses $[15,16]$. Relief agencies train low-skilled community health workers (CHWs) largely as health promoters and instruct them to recommend referral for children needing antibiotic treatment [15]. However, CHW referral recommendations often do not lead to action even in stable settings, and CHWs unable to provide medication often lack credibility [12]. Indeed, even trained health workers often fail to convince families to take a child with severe complications to hospital in a timely manner $[17,18]$. Insecurity reinforces those factors that prevent many families from seeking care in stable environments; financial resources shrink, security concerns increase, travel is restricted, and heightened social tensions may change perceptions of the acceptability of seeking care from particular facilities or individuals. Relief agencies, donors, ministries of health, and other health actors should therefore consider how community case management of childhood pneumonia may compare with mobile clinics and other strategies they employ to reduce child mortality in hard-to-access populations.

This paper aims to generate quantitative evidence for health policy regarding the relative effectiveness of two alternative strategies, mobile clinics and CHWs in fixed posts, for antibiotic treatment of childhood pneumonia in hard-to-access populations. It develops a mathematical decision model that reflects both clinical aspects of pneumonia case management and practical aspects of implementing each intervention in order to provide recommendations to humanitarian agencies, donors, and other key decision-makers.

\section{Methods}

A decision analytic model was developed according to the stages recommended by Briggs et al by specifying the decision problem and boundaries of analysis, structuring the decision model, identifying appropriate evidence, and dealing with uncertainty and heterogeneity [19].

\section{Decision problem}

The study population was identified as a hypothetical cohort of children aged 1-59 months dispersed across a high-mortality, remote setting, where antibiotic treatment for pneumonia is currently unavailable. No geographic location was specified.

Outcomes for this hypothetical cohort were examined under the following three scenarios:

1) No pneumonia treatment available;

2) Mobile clinics are present in the community on an intermittent basis, providing highly accurate diagnosis and treatment when they are present and no treatment when they are absent;

3) A CHW is paid and supported to diagnose and treat pneumonia with antibiotics in the community; while living in the community and therefore available at virtually any time in locations as accessible (if not more so) than mobile clinics, the CHW provides less accurate diagnosis and treatment of pneumonia than the mobile clinic staff.

The model predicts the cumulative number of pneumonia deaths over a given period. Accordingly, the comparison between the two strategies is expressed as the relative rate of death from pneumonia under either strategy, compared to no treatment. While reducing the 
number of cases that become severe and the duration of disease may also improve nutritional status, development and educational attainment, and generally mitigate other untoward outcomes [20], these potential secondary benefits were not included in the model.

\section{Model structure}

We adopted a static rather than transmission dynamic model, as the strategies evaluated are likely to have a negligible impact on disease transmission. The vast majority of children in developing countries are infected with Streptococcus pneumoniae, Haemophilus influenzae type $\mathrm{B}$ and other aetiologic agents of life-threatening pneumonia: such nasopharyngeal carriage is mostly asymptomatic and correlates poorly with invasive disease $[21,22]$. The incidence of pneumonia seems to depend mainly on host and environmental factors [22,23].

Using Microsoft Excel Visual Basic [24], we developed a modified Markov cycle tree model $[19,25]$ to reflect the stochastic nature of and uncertainty in various processes determining childhood pneumonia outcome under each of the scenarios. Markov models are characterised by a finite number of discrete health states, with individuals facing a probability of transition from one health state to another at the end of each of a series of discrete time steps (in our case days). The model was implemented over a time horizon of one hundred days, which reflects the short-term nature of humanitarian programme implementation.

\section{Health states}

The six health states are healthy (meaning not ill with pneumonia), non-severe pneumonia, non-severe pneumonia under treatment, severe pneumonia, severe pneumonia under treatment, and death (Figure 1). Children enter the model in the healthy state, and are exposed to a constant pneumonia incidence rate, which reflects our assumption that case management will not affect incidence.

\section{Tunnel states and memory}

The so-called "Markov assumption" or "memoryless" aspect of the traditional Markov model was relaxed by creating "tunnel states." This approach built temporary memory into the model by tracking the first five days spent with pneumonia. For example, a healthy child who becomes ill with non-severe pneumonia for two days and then develops severe pneumonia on the third day moves through the health states "non-severe day 1," "non-severe day 2," and "severe day 3" (Additional file 1 ). Incorporating memory into the model allowed for the calculation of time-dependent rates of care-seeking described below.

\section{Transition probabilities}

Figure 2 depicts the possible daily transitions for a healthy child. If a child develops pneumonia, health care is available and the child's caregiver seeks treatment within the same day, the child may transition directly from the healthy to the non-severe or severe treatment states. The decision tree is virtually identical for children starting the day in a non-healthy state, except that the transitions for a child with severe pneumonia are restricted to remaining severe, recovery or death. Decision trees and equations used to calculate transition probabilities are provided in Additional file 1.

\section{Care-seeking behaviour}

Studies indicate that the rate of care seeking is timedependent, with no reported differences between nonsevere and severe pneumonia [15,26-28]. Accordingly,
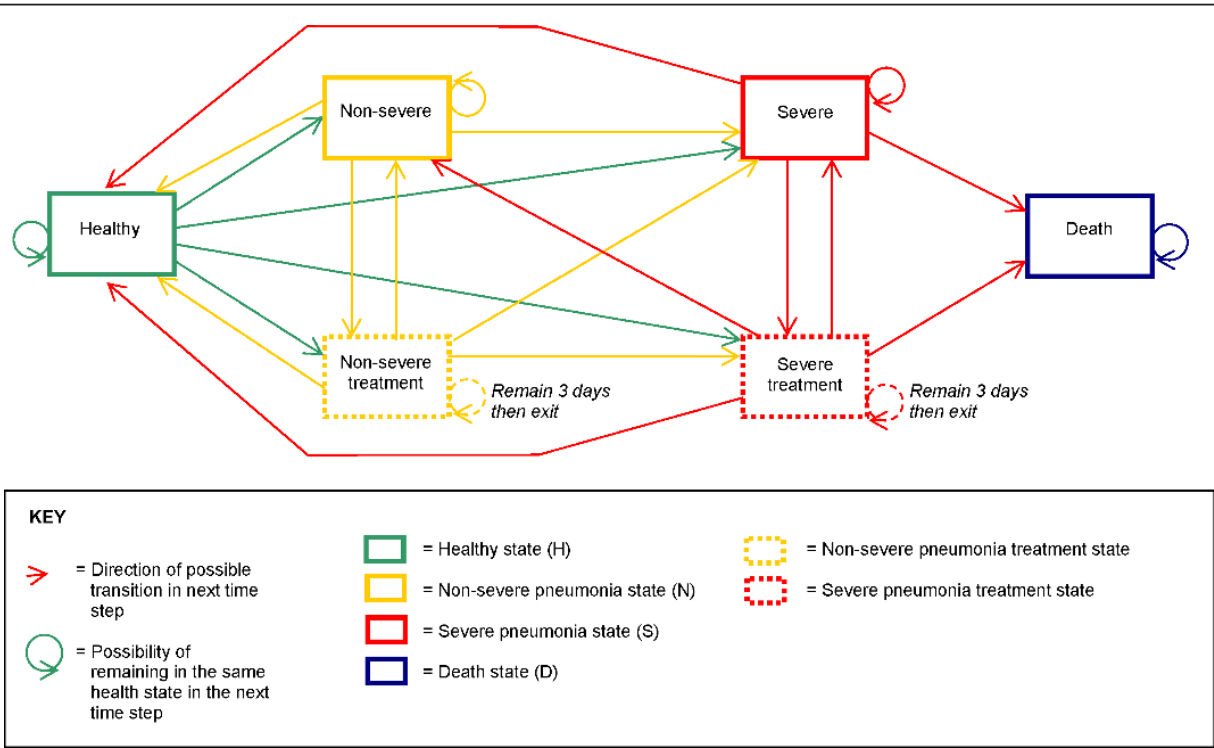

Figure 1 Simplified model structure. 


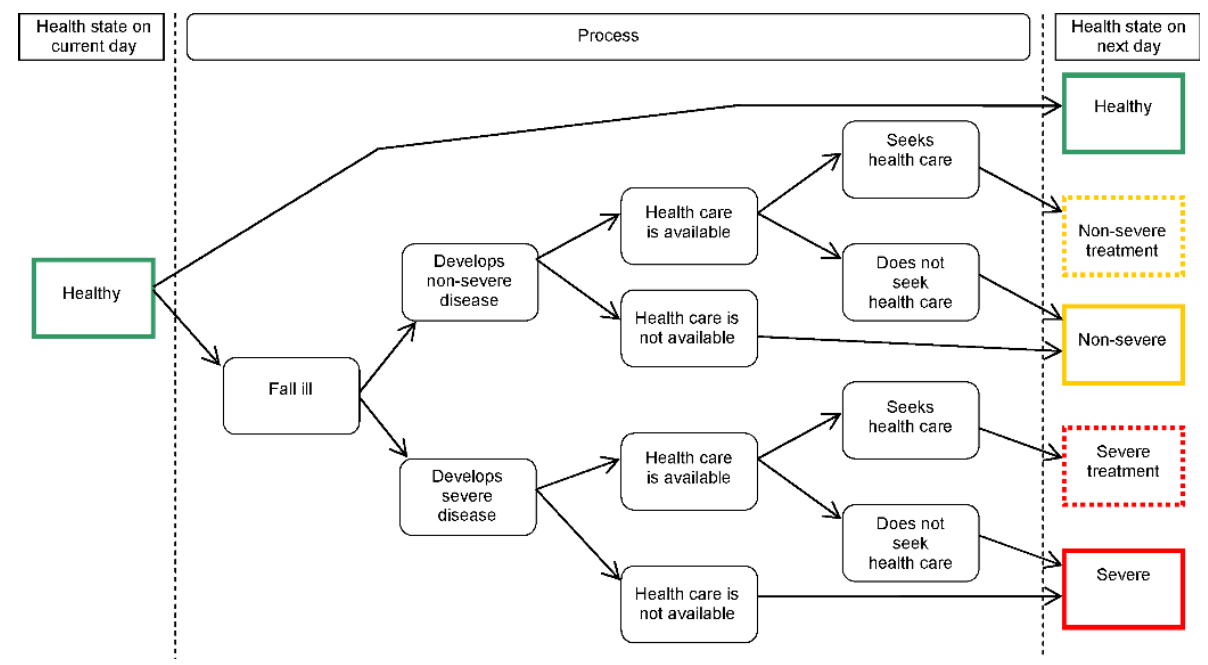

Figure 2 Decision tree: transitions from the healthy state. Healthy children may remain healthy or transition to any of the disease states on the following day. If a child develops pneumonia and health care is both available and sought, the child transitions directly to treatment for pneumonia; otherwise, the child transitions to a disease state (non-severe or severe pneumonia) without treatment. Values and equations defining transition probabilities are detailed in the Appendix.

the daily probability of seeking care was modelled as a function of how many days the child had spent with pneumonia, irrespective of severity. Available evidence was used to calculate the relative rate of care seeking for each day a child may have pneumonia, up to a maximum of five days, beyond which data are not available and the relative rates are therefore modelled as remaining constant.

\section{Parameter values and sensitivity analyses}

Parameter values were informed by evidence from the literature and expert advice. Ethical approval was obtained for expert interviews from the London School of Hygiene and Tropical Medicine. PubMed, EMBASE and Google Scholar were searched for key terms and authors; literature recommendations were sought during expert interviews; and the bibliographic trail of relevant references was followed to exhaustion. Sources of parameter values are provided in Additional file 1, and the values of key parameters are shown in Table 1.

To account for uncertainty in parameter values, we implemented the model stochastically. Accordingly, beta and lognormal probability distributions for each parameter were constructed following the standard methods proposed by Briggs et al [19], ensuring that the distributions reflected reasonable beliefs about the parameter and were defined over an appropriate interval. The standard error for a parameter value was set to five percent of the mean value of that parameter [29]. We then ran 10,000 iterations of the model, sampling randomly from each parameter distribution at each time step.
We also performed sensitivity analyses to explore how results would vary according to pneumonia incidence rate (the sensitivity range of 0.3 to 1.9 pneumonia episodes per child-year was based on Rudan et al [30]: the lower limit corresponds with Rudan's estimate of the median in developing countries, while the upper limit corresponds to the maximum value recorded in $28 \mathrm{com}$ munity-based longitudinal studies that met quality criteria); the accuracy of triage; the frequency of mobile clinic visits; and care-seeking behaviour.

\section{Results \\ Validity}

In the no treatment scenario, the model predicted a pneumonia-specific mortality rate of 0.51 (95\% credible interval or CI: 0.49 to 0.54 ) pneumonia deaths per 10,000 child-days, or 18.62 (95\% CI: 17.89 to 19.71) deaths per 1000 child-years. Assuming pneumonia causes $23 \%$ of child mortality [31], this translates to an all-cause mortality rate of 2.24 deaths per 10,000 childdays or 71.25 deaths per 1000 child-years. Such rates are consistent with the high mortality settings addressed in this paper, as the commonly agreed thresholds defining a humanitarian emergency are 2.1 deaths in SubSaharan Africa or 1.7 deaths in least developed countries per 10,000 child-days [32].

\section{Baseline analysis}

For the mobile clinic strategy, baseline analysis predicted a median pneumonia-specific mortality rate of 0.45 deaths per 10,000 child-days ( $95 \%$ CI: 0.43 to 0.48 ), or a 
Table 1 Values of key model parameters in the baseline analysis

\begin{tabular}{|c|c|c|c|c|c|}
\hline \multirow[t]{2}{*}{ Parameter } & \multicolumn{3}{|c|}{ Scenario } & \multirow[t]{2}{*}{ Distribution } & \multirow[t]{2}{*}{ Source } \\
\hline & $\begin{array}{l}\text { No } \\
\text { treatment }\end{array}$ & Mobile clinic & $\begin{array}{l}\text { Community Health } \\
\text { Worker }\end{array}$ & & \\
\hline $\begin{array}{l}\text { Incidence of pneumonia } \\
\text { (episodes per child per year) }\end{array}$ & 0.7 & 0.7 & 0.7 & Beta & Rudan et al. [30] \\
\hline Care-seeking behaviour & $\mathrm{n} / \mathrm{a}$ & \multicolumn{2}{|c|}{$\begin{array}{c}\text { Median duration before care sought }=3 \\
\text { days, Cumulative probability of seeking care } \\
=90 \%\end{array}$} & Lognormal & $\begin{array}{c}\text { Kallander et al [26] } \\
\text { Sodemann et al } \\
{[45]}\end{array}$ \\
\hline $\begin{array}{l}\text { Probability that treatment is available on any given } \\
\qquad \text { day }\end{array}$ & $0 \%$ & $\begin{array}{l}100 \% \text { on day of } \\
\text { weekly visit, } \\
0 \% \text { on other days }\end{array}$ & $100 \%$ & $\mathrm{n} / \mathrm{a}$ & $\begin{array}{l}\text { Assumption of the } \\
\text { model }\end{array}$ \\
\hline Probability of correct diagnosis and prescription & $\mathrm{n} / \mathrm{a}$ & $90 \%$ & $80 \%$ & Beta & $\begin{array}{l}\text { Kallander et al [27] } \\
\text { Dawson et al [12] } \\
\text { Lim et al [42] }\end{array}$ \\
\hline Probability of adherence to treatment & $\mathrm{n} / \mathrm{a}$ & & $0 \%$ & Beta & Checchi et al [41] \\
\hline $\begin{array}{l}\text { Probability that treatment for non-severe pneumonia } \\
\text { is efficacious }\end{array}$ & $\mathrm{n} / \mathrm{a}$ & & $5 \%$ & Beta & $\begin{array}{l}\text { Hazir et al [13] } \\
\text { Lim et al [42] }\end{array}$ \\
\hline $\begin{array}{c}\text { Probability that treatment for severe pneumonia is } \\
\text { efficacious }\end{array}$ & $\mathrm{n} / \mathrm{a}$ & $90 \%$ & $80 \%$ & Beta & $\begin{array}{c}\text { Kabra et al [14] } \\
\text { Lim et al [42] } \\
\text { Zaman et al [46] } \\
\text { Johnson et al [47] } \\
\text { Hazir et al [13] } \\
\text { Banajeh et al [48] }\end{array}$ \\
\hline
\end{tabular}

reduction of $12.1 \%$. The $\mathrm{CHW}$ strategy produced a lower median rate of 0.31 deaths per 10,000 child-days (95\% CI: 0.29 to 0.32 ), or a $40.5 \%$ reduction. This latter figure is comparable to the pneumonia-specific mortality reductions measured in CHW projects in Uganda by Kallander et al [27] and in Nepal by Dawson et al [12]. While all iterations of the mobile clinic strategy resulted in mortality reductions with respect to no treatment, all iterations of the CHW strategy resulted in greater mortality reductions than the mobile clinic strategy. The CHW strategy is therefore superior to a weekly mobile clinic under baseline assumptions.

\section{Sensitivity analyses}

As pneumonia incidence increased, the CHW strategy achieved increasingly greater mortality reductions than the mobile clinic strategy (Figure 3). However, mobile clinics that visit every day achieved greater mortality reductions than the baseline CHW strategy (Figure 4), as would be expected given the former's higher diagnostic accuracy and lower case-fatality ratio (CFR) for severe cases. If the mobile clinic were to diagnose and correctly prescribe treatment for pneumonia with $100 \%$ sensitivity, it would achieve a $12 \%$ greater pneumoniaspecific mortality reduction than under the baseline assumption of $90 \%$ sensitivity (Figure 5). If the CHW strategy were only to diagnose and prescribe treatment correctly for $40 \%$ of cases seen rather than the baseline assumption of $80 \%$, it would achieve a $52 \%$ lower mortality reduction of 0.1 deaths per 10,000 child-days, which would be approximately equivalent to a mobile clinic with $90 \%$ sensitivity visiting every four days (Figure 4).

Assumptions regarding the time-dependent nature of care-seeking were also explored. Caregivers who know on which days the mobile clinic will visit may adapt their care-seeking behaviour; instead of depending on time since illness onset, care-seeking may simply be a binary probability on the day of clinic visit. Figure 6 shows that the mortality reduction achieved by a weekly mobile clinic would be comparable to those of a $\mathrm{CHW}$ if approximately $70 \%$ of children with any severity of pneumonia onset were taken to the mobile clinic and the mobile clinic were able to consult all cases. In addition, if all caregivers who would have sought care on a day prior to the mobile clinic's visit did so on the day of the visit (and whose careseeking behaviour was therefore a cumulative time-dependent probability), the mobile clinic would reduce mortality by 0.17 deaths per 10,000 child-days (CI: 0.15 to 0.19 ) and achieve a $32.8 \%$ reduction in pneumonia-specific mortality.

\section{Discussion}

We find that a $\mathrm{CHW}$ in a fixed health post would achieve greater mortality reductions than a weekly mobile clinic strategy in the majority of sensitivity analyses conducted. In univariate sensitivity analyses, mobile clinics achieved greater mortality reductions where the probability of seeking care from a mobile clinic was significantly higher than from a $\mathrm{CHW}$, and where mobile clinics visited communities daily. Mobile clinics would also achieve greater mortality reductions 


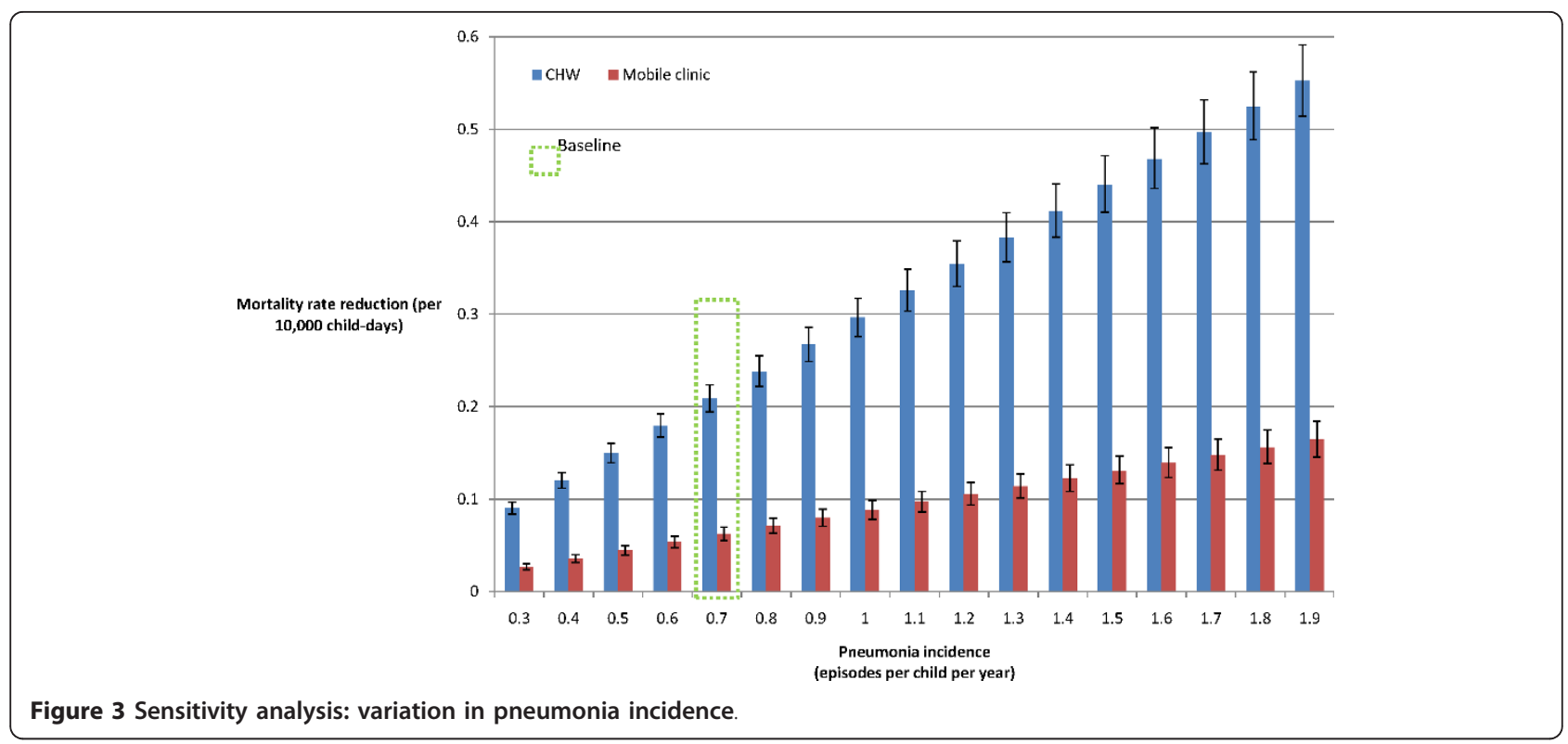

than the CHW strategy if the mobile clinic had 100\% sensitivity to diagnose and treat children while the CHW strategy had approximately $30 \%$ or lower sensitivity, or if a number of variables simultaneously differed substantially from our baseline assumptions. For a number of important parameters, only limited evidence is available and parameter values may differ between contexts. These findings therefore do not conclusively show one strategy to be more effective than the other. Rather, current evidence indicates that a CHW in a fixed post is a viable alternative to mobile clinics, and therefore warrants consideration in high-mortality, hard-to-access areas.

In line with humanitarian decision-making, this model focussed on the short term impact of the alternative strategies; however, the longer term impact on health and health systems should also be taken into account. Mobile clinics may be quicker to implement in response

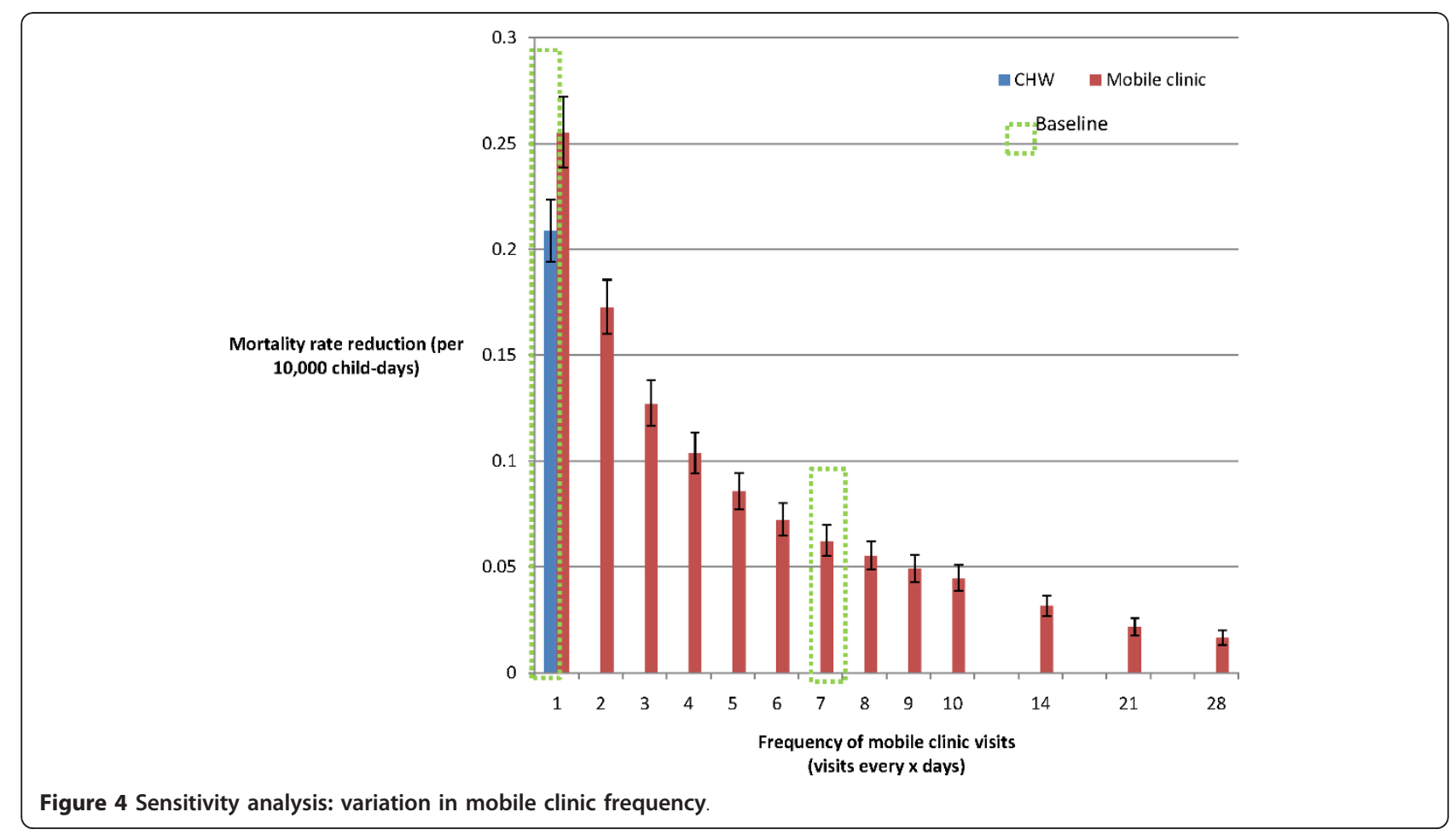




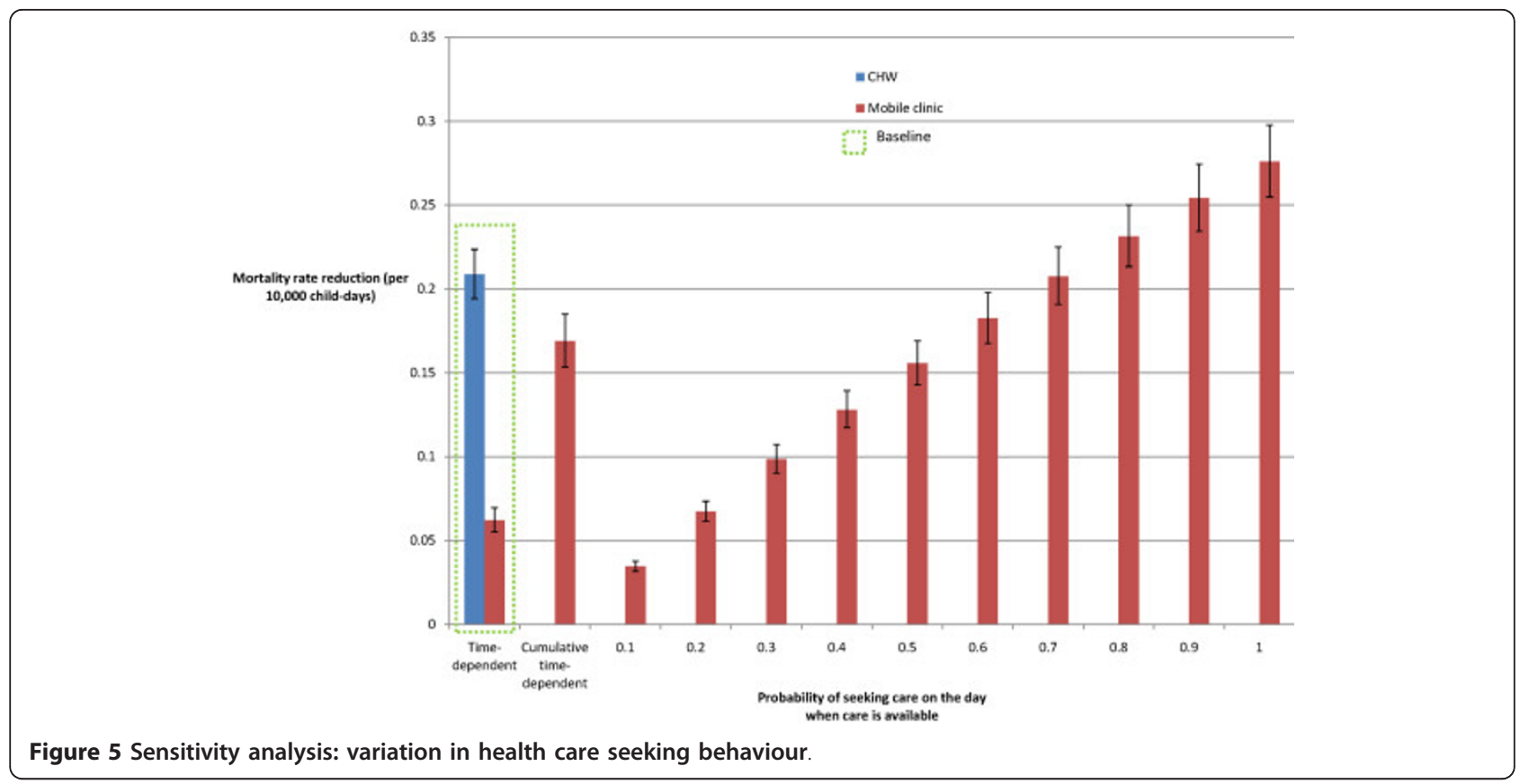

to a sudden-onset crisis, but they may also risk creating dependence and undermining community coping strategies [33]. Supporting CHWs may foster sustainable local development and be more readily integrated into the public health system, but identifying potential CHWs is not always straightforward [34], especially when social tensions are high and different individuals are acceptable to different groups. Both strategies rely upon effective and reliable drugs supply and CHWs especially require regular supervision and support to be effective $[12,34]$.

Agencies with the resources to respond quickly to an emerging crisis by deploying mobile clinics should consider initiating a transition to community-based treatment from their very first visit by identifying existing or potential CHWs to train. The role of the mobile clinic

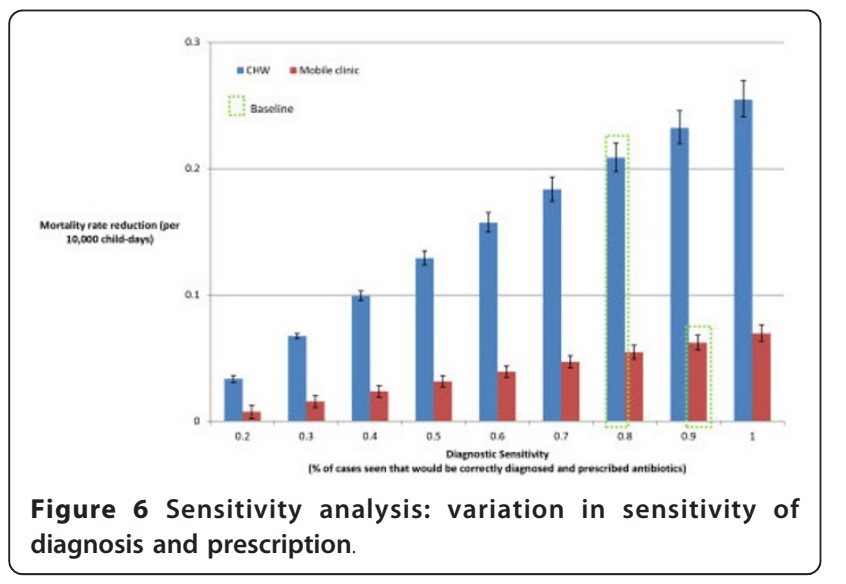

can then evolve into providing regular high-quality supplies and supervision to CHWs. With preparation, this supportive approach can also ensure that treatment remains available even if insecurity or other obstacles prevent the mobile team from visiting for a period. By supporting CHWs to treat pneumonia and other acute diseases, the mobile team could potentially also use its visits to engage in preventive interventions, such as immunizations, and to provide specialized treatment for more chronic conditions.

By providing a direct quantitative comparison of the health impact of these two strategies for reaching hardto-access populations, this paper responds to calls from leaders in epidemiology and public health for research into implementation strategies in high-mortality settings $[1,35,36]$. Modelling can be a useful tool to foster rational decision-making based on explicit assumptions and scientific evidence for hard-to-access populations in low-income areas. While computationally intensive, the model is transparent and intuitive, and the decision analytic approach is adapted to the needs of decisionmakers. As new data comes to light, parameter values can be changed and aspects of the model can be refined.

In quantifying the uncertainty in the decisions, the model also highlights the need for humanitarian and development programmes to undertake research in hard-to-access areas to reduce decision uncertainty. Improving the evidence base would require focussed data collection on parameter values from a given setting where both strategies are implemented or formal comparisons of the two approaches, although the latter 
could potentially have untenable ethical implications. Cost-effectiveness analyses would offer a more complete evidence base for deciding between the two strategies, as even in emergency interventions, efficiency is required to maximize the impact of the finite human, logistic, and financial resources that can be mobilized. Although the relative costs of the interventions are likely to vary considerably according to the specificities of each context, in general mobile clinics have been a resource-intensive intervention [10]; this suggests that a $\mathrm{CHW}$ strategy will usually be the more cost-effective option of the two if local contextual parameters indicate that the two strategies would have similar curative effectiveness.

This model can and should also be extended to the treatment of other key childhood diseases. In addition to diarrhoea and malaria, neonatal deaths constitute a significant proportion of under five mortality and many are attributable to invasive bacterial infections, including pneumonia, sepsis, and meningitis, which can be treated with antibiotics $[2,35,37,38]$. Sazawal et al provide some evidence for community management of neonatal pneumonia [11], and a number of countries have begun to include protocols for neonatal care within IMCI guidelines [39], but further research into community case management of neonates, especially in hard-to-access areas, is likely to offer a significant contribution to public health.

As many programmes have turned towards CHWs to increase coverage of their interventions, it has become clear that CHWs cannot do everything, especially if they are unpaid $[34,40]$. Detailed national and sub-national analysis is required to understand how integrated community-based approaches can be implemented in high mortality hard-to-access areas [38], including those affected by instability.

\section{Limitations}

By its very nature, modelling entails simplifying reality to make useful predictions. Some important aspects of childhood pneumonia were excluded because they were not expected to have a significant impact on the relative effectiveness of the two strategies being compared. Certain unexplored issues may, however, have a more significant influence on the results. Sensitivity analysis was not conducted to explore the responsiveness of the outcome to changes in the median duration of disease, or how treatment adherence may differ between mobile clinics and fixed health posts. Analysis of alternative models of care-seeking behaviour revealed the possibility that mobile clinics may be the dominant strategy when attendance is very high and the mobile clinic is able to consult all patients who attend. In practice, mobile clinics may not be able to see all patients and so the degree to which these findings hold true will depend on the effectiveness of triage. Insecurity or other constraints may also cause a mobile team to miss planned visits, which, in addition to reducing this strategy's impact, may potentially also have untoward consequences for care-seeking practices, although evidence on this latter point is not currently available. Similarly, we assumed that CHWs would be available $100 \%$ of the time, and did not model the potential repercussions of the CHW running out of antibiotics or being unavailable. Estimating the true effect of either intervention would also require considering simultaneous diagnosis (or misdiagnosis) and treatment of co-morbidities, such as malaria.

Standard errors used for sensitivity analysis were estimated somewhat crudely and may therefore have underestimated the uncertainty in the results. All children began the model in the healthy state and a burn-in period was not used, which likely biased the expected number of child deaths downwards. While sensitivity analysis was conducted to examine the impact on results of different pneumonia incidences, outcomes were not examined in contexts of heightened vulnerability, such as would accompany high malnutrition or HIV rates, where the rate of disease progression and the CFR would increase [41]. Perhaps most influentially, the model does not measure the negative impact of unnecessary antibiotic treatment, incorrect prescriptions, and poor adherence on the individual child or on the wider community, particularly with respect to the development of resistant bacterial strains. Lim provides an example of how such negative outcomes can be included in a decision tree [42].

Like most studies, this analysis did not formally address methodological and model uncertainty, which Brisson and Edmunds have shown can play an even greater role than parameter uncertainty [43]. Parameter estimates themselves were based on a non-systematic review of the literature and the Delphi method was not used to elicit expert opinion on parameter values, although this method also has drawbacks [44]. The number of relevant studies with quantitative data on certain topics, such as care-seeking behaviour and treatment adherence in crises, was limited. While some grey literature was obtained, studies and reports, particularly from within the humanitarian community may have been missed. A broader limitation of this project is the lack of rigorous data collected by humanitarian agencies and ministries of health working in hard-to-reach areas.

\section{Conclusions}

This paper's findings indicate that to reduce high under 5 mortality rates through curative care in hard- 
to-access populations, humanitarian health agencies, donors, and ministries of health should work together to:

- ensure that mobile clinic strategies explicitly consider how the timing of community visits, care-seeking behaviour, the type of intervention (preventive, curative for slow-onset illness, curative for rapidonset illness), and other key variables identified in this model can be expected to influence the strategy's health impact;

- consider how training and support of CHWs can be integrated into the activities of a mobile team with high-level technical skills and resources;

- advocate for and design policies and programmes that enable CHWs to provide antibiotics at the community level;

- use models to guide health policy and explicit decision-making, especially for hard-to-access areas where current evidence is limited;

- prioritise research into care-seeking behaviour, treatment adherence, rates of disease progression and other key factors in service delivery strategies.

\section{Additional material}

Additional file 1: Appendix. Appendix containing details on the model.

\section{List of abbreviations}

(CFR): Case-fatality ratio; (CHW): Community health worker; (Cl): Credible interval; (FCHV): female community health volunteers; (MDG): Millennium development goal.

\section{Acknowledgements}

We are grateful for fruitful discussions with Brian Greenwood (LSHTM), Karen Edmond (LSHTM), Rudi Coninx (WHO), Stéphane du Mortier (International Committee of the Red Cross), and several other field workers experienced in working with mobile clinics and supporting CHWs. This study was carried out without sources of funding.

\section{Author details}

${ }^{1}$ Department of Global Health \& Development, London School of Hygiene \& Tropical Medicine, 15-17 Tavistock Place, London WC1H 9SH, UK. ${ }^{2}$ Department of Health Services \& Policy Research, London School of Hygiene \& Tropical Medicine, 15-17 Tavistock Place, London WC1H 9SH, UK. ${ }^{3}$ Department of Disease Control, London School of Hygiene \& Tropical Medicine, Keppel Street, London WC1E 7HT, UK.

\section{Authors' contributions}

CP designed the study, developed the model, carried out analyses and cowrote the paper. BR supervised the study and co-wrote the paper. FC designed and supervised the study and co-wrote the paper. All authors read and approved the final manuscript.

\section{Competing interests}

The authors declare that they have no competing interests.

Received: 11 June 2011 Accepted: 10 January 2012

Published: 10 January 2012
References

1. Mulholland E, Smith L, Carneiro I, Becher H, Lehmann D: Equity and childsurvival strategies. Bull World Health Organ 2008, 86:399-407.

2. Bhutta ZA, Chopra M, Axelson H, Berman P, Boerma T, Bryce J, Bustreo F, Cavagnero E, Cometto G, Daelmans B, et al: Countdown to 2015 decade report (2000-10): taking stock of maternal, newborn, and child survival. Lancet 2010, 375:2032-2044.

3. Salama P, Spiegel $P$, Talley L, Waldman R: Lessons learned from complex emergencies over past decade. Lancet 2004, 364:1801-1813.

4. Black RE, Morris SS, Bryce J: Where and why are 10 million children dying every year? Lancet 2003, 361:2226-2234.

5. Connolly MA, Gayer M, Ryan MJ, Salama P, Spiegel P, Heymann DL: Communicable diseases in complex emergencies: impact and challenges. Lancet 2004, 364:1974-1983.

6. du Mortier S, Coninx R: Mobile health units in emergency operations: a methodological approach. Humanitarian Practice Network Paper 2007.

7. Shaw AP, Cattand P: Analytical tools for planning cost-effective surveillance in Gambiense sleeping sickness. Med Trop (Mars) 2001, 61:412-421.

8. Chippaux JP, Boussinesq M, Prod'hon J: [The use of ivermectin in the control of onchocerciasis]. Sante 1995, 5:149-158.

9. Amazigo U, Noma M, Boatin BA, Etya'ale DE, Seketeli A, Dadzie KY: Delivery systems and cost recovery in Mectizan treatment for onchocerciasis. Ann Trop Med Parasitol 1998, 92(Suppl 1):S23-31.

10. du Mortier S, Coninx R: Mobile health units in emergency operations: a methodological approach. Book Mobile health units in emergency operations: a methodological approach City: Humanitarian Practice Network; 2007.

11. Sazawal S, Black RE: Effect of pneumonia case management on mortality in neonates, infants, and preschool children: a meta-analysis of community-based trials. Lancet Infect Dis 2003, 3:547-556.

12. Dawson P, Pradhan Y, Houston R, Karki S, Poudel D, Hodgins S: From research to national expansion: 20 years' experience of communitybased management of childhood pneumonia in Nepal. Bull World Health Organ 2008, 86:339-343.

13. Hazir T, Fox LM, Nisar YB, Fox MP, Ashraf YP, MacLeod WB, Ramzan A, Maqbool S, Masood T, Hussain W, et al: Ambulatory short-course highdose oral amoxicillin for treatment of severe pneumonia in children: a randomised equivalency trial. Lancet 2008, 371:49-56.

14. Kabra SK, Lodha R, Pandey RM: Antibiotics for community acquired pneumonia in children. Cochrane Database Syst Rev 2006, 3:CD004874.

15. Moss WJ, Ramakrishnan M, Storms D, Henderson Siegle A, Weiss WM, Lejnev I, Muhe L: Child health in complex emergencies. Bull World Health Organ 2006, 84:58-64.

16. Medecins Sans Frontieres: Clinical guidelines: Diagnosis and treatment manual for curative programmes in hospitals and dispensaries: guidance for prescribing. 7th revised edition. Paris; 2007.

17. Cook J, Amevigbe PM, Crost M, Gbetoglo D, Tursz A, Assimadi JK: [Health care seeking behavior of children in Togo]. Rev Epidemiol Sante Publique 1999, 47(Suppl 2):2S93-113.

18. Bang AT, Bang RA, Morankar VP, Sontakke PG, Solanki JM: Pneumonia in neonates: can it be managed in the community? Arch Dis Child 1993, 68:550-556.

19. Briggs AH, Claxton K, Sculpher MJ: Decision modelling for health economic evaluation Oxford: Oxford University Press; 2006.

20. Smith TA, Lehmann D, Coakley C, Spooner V, Alpers MP: Relationships between growth and acute lower-respiratory infections in children aged less than $5 \mathrm{y}$ in a highland population of Papua New Guinea. Am J Clin Nutr 1991, 53:963-970.

21. Mulholland K: Strategies for the control of pneumococcal diseases. Vaccine 1999, 17(Suppl 1):S79-84.

22. Rudan I, Boschi-Pinto C, Biloglav Z, Mulholland K, Campbell H: Epidemiology and etiology of childhood pneumonia. Bull World Health Organ 2008, 86:408-416.

23. Mulholland K: Perspectives on the burden of pneumonia in children. Vaccine 2007, 25:2394-2397.

24. Visual Basic 6.5. Book Visual Basic 6.5 City: Microsoft Corporation; 2006.

25. Hollenberg J: Markov Cycle Trees: A New Representation for Complex Markov Processes. Abstract from the Sixth Annual Meeting of the Society for Medical Decision Making. Med Decis Making 1984, 4. 
26. Kallander K, Hildenwall H, Waiswa P, Galiwango E, Peterson S, Pariyo G: Delayed care seeking for fatal pneumonia in children aged under five years in Uganda: a case-series study. Bull World Health Organ 2008, 86:332-338.

27. Kallander K, Tomson G, Nsabagasani X, Sabiiti JN, Pariyo G, Peterson S: Can community health workers and caretakers recognise pneumonia in children? Experiences from western Uganda. Trans $R$ Soc Trop Med Hyg 2006, 100:956-963.

28. Aung T, Tun KM, Thinn K, Thein AA: Knowledge, attitudes and practices of mothers on childhood acute respiratory infections (ARI). Southeast Asian J Trop Med Public Health 1994, 25:590-593.

29. Critchfield GC, Willard KE: Probabilistic analysis of decision trees using Monte Carlo simulation. Med Decis Making 1986, 6:85-92.

30. Rudan I, Tomaskovic L, Boschi-Pinto C, Campbell H: Global estimate of the incidence of clinical pneumonia among children under five years of age. Bull World Health Organ 2004, 82:895-903.

31. Williams BG, Gouws E, Boschi-Pinto C, Bryce J, Dye C: Estimates of worldwide distribution of child deaths from acute respiratory infections. Lancet Infect Dis 2002, 2:25-32.

32. The Sphere Project: Humanitarian charter and minimum standards in humanitarian response. 3 edition. Rugby, UK: Practical Action Publishing; 2011.

33. Van Damme WI, Van Lerberghe WI, Boelaert M: Primary health care vs. emergency medical assistance: a conceptual framework. Health Policy Plan 2002, 17:49-60.

34. Haines A, Sanders D, Lehmann U, Rowe AK, Lawn JE, Jan S, Walker DG, Bhutta Z: Achieving child survival goals: potential contribution of community health workers. Lancet 2007, 369:2121-2131.

35. Rudan I, El Arifeen S, Black RE, Campbell H: Childhood pneumonia and diarrhoea: setting our priorities right. Lancet Infect Dis 2007, 7:56-61.

36. Greenwood B: A global action plan for the prevention and control of pneumonia. Bull World Health Organ 2008, 86:322-322A.

37. Black RE, Cousens S, Johnson HL, Lawn JE, Rudan I, Bassani DG, Jha P, Campbell H, Walker CF, Cibulskis R, et al: Global, regional, and national causes of child mortality in 2008: a systematic analysis. Lancet 2010, 375:1969-1987.

38. Wardlaw T, Salama P, Johansson EW, Mason E: Pneumonia: the leading killer of children. Lancet 2006, 368:1048-1050.

39. Bryce J, Harris Requejo J: Countdown to 2015: Tracking progress in maternal, newborn, and child survival: The 2008 Report. Book Countdown to 2015: Tracking progress in maternal, newborn, and child survival: The 2008 Report City.

40. Van Lerberghe W, Conceicao C, Van Damme W, Ferrinho P: When staff is underpaid: dealing with the individual coping strategies of health personnel. Bull World Health Organ 2002, 80:581-584.

41. Checchi F, Gayer M, Grais R, Mills E: Public health in crisis-affected populations: A practical guide for decision-makers, Network Paper 61. In Book Public health in crisis-affected populations: A practical guide for decisionmakers, Network Paper 61. Volume 61. City: Humanitarian Practice Network at the Overseas Development Institute; 2007.

42. Lim YW, Steinhoff M, Girosi F, Holtzman D, Campbell H, Boer R, Black R, Mulholland K: Reducing the global burden of acute lower respiratory infections in children: the contribution of new diagnostics. Nature 2006, 444(Suppl 1):9-18.

43. Brisson M, Edmunds WJ: Impact of model, methodological, and parameter uncertainty in the economic analysis of vaccination programs. Med Decis Making 2006, 26:434-446.

44. Hutchings A, Raine R, Sanderson C, Black N: A comparison of formal consensus methods used for developing clinical guidelines. $J$ Health Serv Res Policy 2006, 11:218-224.

45. Sodemann M, Jakobsen MS, Molbak K, Alvarenga IC Jr, Aaby P: High mortality despite good care-seeking behaviour: a community study of childhood deaths in Guinea-Bissau. Bull World Health Organ 1997, 75:205-212.

46. Zaman K, Baqui AH, Yunus M, Sack RB, Bateman OM, Chowdhury HR, Black RE: Acute respiratory infections in children: a community-based longitudinal study in rural Bangladesh. J Trop Pediatr 1997, 43:133-137.

47. Johnson AW, Osinusi K, Aderele WI, Adeyemi-Doro FA: Bacterial aetiology of acute lower respiratory infections in pre-school Nigerian children and comparative predictive features of bacteraemic and non-bacteraemic illnesses. J Trop Pediatr 1993, 39:97-106.
48. Banajeh SM: Outcome for children under 5 years hospitalized with severe acute lower respiratory tract infections in Yemen: a 5 year experience. J Trop Pediatr 1998, 44:343-346.

\section{Pre-publication history}

The pre-publication history for this paper can be accessed here: http://www.biomedcentral.com/1472-6963/12/9/prepub

doi:10.1186/1472-6963-12-9

Cite this article as: Pitt et al:: Treating childhood pneumonia in hard-toreach areas: $A$ model-based comparison of mobile clinics and community-based care. BMC Health Services Research 2012 12:9.

\section{Submit your next manuscript to BioMed Central and take full advantage of:}

- Convenient online submission

- Thorough peer review

- No space constraints or color figure charges

- Immediate publication on acceptance

- Inclusion in PubMed, CAS, Scopus and Google Scholar

- Research which is freely available for redistribution

Submit your manuscript at www.biomedcentral.com/submit
Biomed Central 